\title{
Optimal and robust digital current controller synthesis for vector-controlled induction motor drive systems
}

\author{
L. Umanand \\ S.R. Bhat
}

Indexing terms: Vector control, Induction motor drives, Current controllers

\begin{abstract}
The vector-controlled induction-motor drive is a multi-input multi-output system. By using modern control techniques, all feedback loops can be closed simultaneously, in addition to obtaining optimal gains for the controllers. A systematic approach to the design of the digital current controllers is proposed. A vectorcontrolled induction-motor-drive system is usually viewed as a full-state feedback problem, where the non-measurable states are estimated using suitable reduced-order observers. Here, the induction-motor-drive system is viewed as a linear quadratic (LQ) tracker problem with output feedback, as this approach uses only the measurable states of the system and provides flexibility in choosing the control structure. The current controllers are designed for the torque dynamics of the induction motor. The controller should also be so designed that the whole drive system is stable for a class of induction motors, i.e. the whole drive system should be stable in the face of uncertainties in the parameters of the induction motor. Therefore, it becomes essential to build stability robustness into the controller design. Here, the proposed systematic approach determines the optimal controller gains for a given performance specification under the constraint that the whole system has stability robustness for uncertainties in the specified parameters of the induction motor. The concepts are illustrated by simulation.
\end{abstract}

\section{Introduction}

Recently, the topic of vector control of inductionmotor drives has drawn considerable attention [1]. The objective of the vector control of induction motors is to independently control the torque and the flux in the motor, so that better dynamic performance, similar to that in a DC motor, is achieved. It can be noted that, in the case of an induction motor, the flux and torque producing components of the stator current are coupled, and hence the dynamic performance is limited. The vector-control technique aims at deriving the torque and the flux producing components of the stator-current space phasor is, to achieve independent control of these components. This is achieved by expressing the stator current as two orthogonal components, the direct (flux producing) $i_{s d}$, and the quadrature (torque producing) $i_{s q}$, components. Further, these two current components are decoupled by aligning the direct component along a synchronously rotating fluxlinkage space phasor. Therefore the vector control is also called field-oriented control.

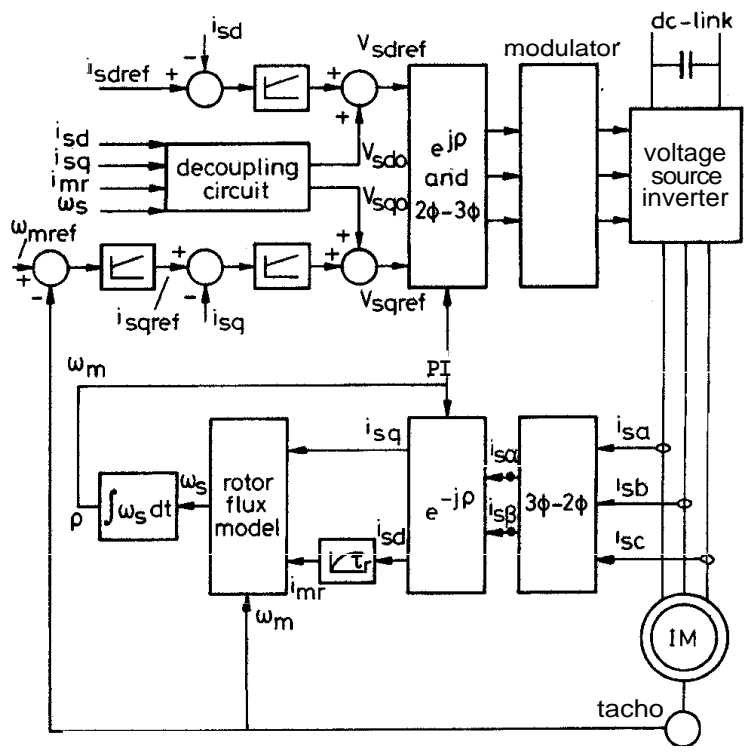

Fig.1 Block schematic of rotor field-oriented control of an induction motor

The block diagram for the rotor field-oriented speed control of an induction motor [2-4], is presented in Fig. 1. The three phase currents $i_{s a}, i_{s b}$ and $i_{s c}$ are measured and transformed into two phase quantities $i_{s \alpha}$ and $i_{s \beta}$. The two phase currents, $i_{s \alpha}$ and $i_{s \beta}$, in the stationary reference frame are transformed to two phase currents, $i_{s d}$ and $i_{s q}$, in a rotating reference frame, which is aligned along the rotor-flux axis. The frame transformation is performed by estimating the rotor-flux position, $\rho$, which uses a rotor-flux model [2-5]. The measured speed, $\omega_{m}$, is compared with the speed reference, and the speed error is passed through a zero 
steady-state-error controller, such as a PI controller, to obtain $i_{\text {sqref }}$. The $i_{\text {sqref }}$ is compared with $i_{s q}$ to obtain the q-axis current error, which is passed through the q-axis current controller. Similarly, $i_{s d}$ is compared with $i_{s d r e f}$, and the error is passed through a d-axis controller. Decoupling terms are added to the d-axis and q-axis controller outputs to obtain $V_{\text {sdref }}$ and $V_{\text {sqref }}[2,5]$. These reference voltages are transformed to the stationary reference frame and passed through the modulator, to appropriately switch the power devices of the voltagesource inverter.

The torque is controlled by the $i_{s q}$ loop, and the flux is controlled by the $i_{s d}$ loop. The speed is controlled by the outer loop. The vector control of an induction motor has to basically tackle two problems: (i) to orient the flux-producing component of the stator-current space phasor along the flux-linkage space phasor, i.e. by estimating the magnitude and position, $p$, of the flux linkage and, (ii) to design current and speed controllers for optimum and robust performance of the induction-motor drive. The problem of field orientation has been extensively discussed [1-5]. This paper aims at the synthesis of optimum and robust current controllers for the induction motor drives.

Traditionally, classical control theory, which is primarily applicable to linear time-invariant systems, was employed for the design of current controllers of the induction motor. The classical control theory is, however, not easy to apply in multi-inputimulti-output (MIMO) systems, such as the induction-motor vectorcontrolled drive system. Due to the interaction of the control loops in a multivariable system, each singleinput/single-output (SISO) transfer function can have acceptable properties in terms of step response and robustness, but the coordinated control motion of the system can fail to be acceptable. Therefore MIMO design using classical techniques requires a painstaking trial and error procedure, using the approach of closing one loop at a time. By using modern control techniques, many of the limitations of the classical controls for multivariable feedback control systems can be overcome. Modern control design is fundamentally a timedomain technique, while classical control design is a frequency-domain technique. A state space model of the system to be controlled is required. The power of modern control has its roots in the fact that the state space model can represent both the MIMO as well as a SISO system [6]. In the context of modern controller synthesis, although the state feedback techniques guarantee closed-loop stability [6,7], all the state components are seldom available for feedback purposes in a practical design problem. As a consequence, the nonavailable state components are estimated from the available states and the system model, using a reducedorder observer. However, the state estimates will depend on the accuracy with which the system parameters (like the rotor and stator resistances in the case of the induction motor) are known. Therefore, to overcome the above problem, the output feedback is used. In the output-feedback approach, only the measurable or the available state components of the system are used for feedback control. Further, unlike the full-state feedback, the output feedback-control law allows any desired dynamical control structure, thereby regaining much of the intuition of classical control design [6]. Nowadays, digital controller synthesis is preferred owing to the availability of powerful and inexpensive digital processors. To achieve the optimum controller, a specific performance criteria has to be minimised. This is usually a linear combination of the quadratics of the states and the inputs of the system. This approach is called the linear quadratic (LQ) approach. In this paper, the background of vector control of induction motor (IM) and optimal control theory is assumed. A summary of the digital LQ tracker with output feedback is given in Appendix 7 for quick reference.

\section{Vector control scheme for IM drive}

The output feedback approach has been extensively used in aircraft applications such as autopilots [8]. In this paper, the output feedback approach will be exploited for control of induction-motor drives. The induction motor can be split into two dynamical systems. One of the systems consists of the speed dynamics, and the other system consists of the torque dynamics for a rotor field-oriented induction-motordrive system. The inner current-control loops, i.e. the $d$-controller and the q-controller, are designed to take care of the torque dynamics, as shown in Fig. 1. The torque dynamics can be analysed, and the current controllers can be synthesised, using the $d-q$ model [2] of the induction motor.

The LQ tracker approach, using output feedback, will be used for synthesising the digital current controllers to obtain the optimal gains. It is a two input-two output system for the current controllers. The optimal gains with guaranteed stability will be obtained, with the added advantage that classical intuition can still be brought forth in the choice of the dynamic control structure for the current controlled system.

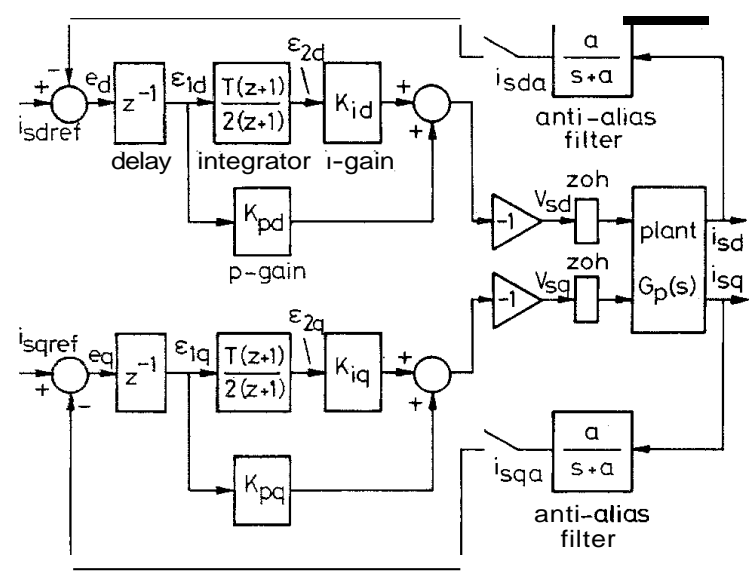

Fig.2 Block schematic for induction-motor-drive system for torque dynamics

The control structure of the d-axis current-control loop $\left(i_{s d}\right.$ loop) and the q-axis current-control loop ( $i_{s q}$ loop) are represented in Fig. 2. In building the plant model of the induction-motor-drive system for the LQ tracker formulation with output feedback, one has to consider the dynamics of the compensator and other delays that are encountered in the control loop. Referring to Fig. 2, the dynamics of the induction-motor model, that is transformed to the discrete domain, and the dynamics of the d-axis and q-axis compensators form a part of the system formulation. The delay due to computation, the inverter delays etc., will also have 
to be taken into account. Rather than exactly calculate the computation and the inverter delays, one can include all delays in the control loop into one sample time. Therefore a sample time delay can be introduced in the d-axis and the q-axis loops, to account for the various delays in the loops, as shown in Fig. 2.

As the rotor-field oriented control is performed using a digital processor, the analogue stator currents should be filtered to remove anti-aliasing effects after sampling. As the anti-alias filter is in the control path, the dynamics of the anti-alias filter should also be included in the system formulation. As the two-phase to threephase and three-phase to two-phase transformations are mere algebraic operations, the dynamics of the induction motor are given by the two phase $d-q$ model $[2,5]$.

The state space model of the induction motor clearly indicates that it is a fourth-order system [2]. However, under field orientation and for the case of constant flux operation, the IM plant reduces to a second-order system. Thus, each of the individual current-control loops basically see a first-order plant. Therefore the compensator structure can be as simple as a PI controller. Such a discrete PI controller is included in Fig. 2.

It is important to note that the performance requirements will be met under the condition of rotor-field orientation. But, if there exists some field disorientation, then the performance criteria, such as speed of response, overshoots, undershoots etc., will not be met. However, the objective should be to synthesise controllers, wherein the stability and performance criteria are met under rotor-field-oriented operation, and, at least, stability criteria should be met when not oriented along the rotor field (wherein the plant is a fourth-order system). Therefore it is important that the stability of the system is maintained, even although there may be loss of performance due to field disorientation.

For the motor specified in Appendix 7.2, the synthesis is carried out to meet the following performance criteria: (i) The steady-state current error should be zero. (ii) The response to a step change in the stator current references (settling time) should be less than $30 \mathrm{~ms}$. (iii) The peak overshoot should be less than $10 \%$. In the following Section, the formulation of the IM drive, as an LQ tracker problem with output feedback, is treated in detail.

\section{Formulation of IM drive as an LQ tracker with output feedback}

In this Section, the mathematical formulation of the IM drive system as a standard LQ tracker with output feedback, in a form as indicated in Appendix 7, is presented. Referring to Fig. 2, the various components, such as the plant dynamics, the anti-alias filter dynamics and the controller dynamics, are described in the following subsections.

\subsection{Plant dynamics}

The induction motor dynamics for torque or current control are given in [2]. Here, the state variables and the input variables $\left(i_{s d}, i_{s q}, \psi_{r d}, \psi_{r q}, V_{s d}\right.$ and $\left.V_{s q}\right)$ are defined to represent deviations in the neighbourhood of an equilibrium point.

The state-space description of the plant is given by

$$
d x / d t=A \mathbf{x}+B u \text { and } \mathrm{y}=C x+D u
$$

where $x$ is the state vector, $u$ is the input vector and $y$ is the output vector. The plant $G_{p}$, is described by matrices $\mathbf{A}_{p}, \mathbf{B}_{p}, \mathbf{C}_{p}$ and $\mathbf{D}_{p}$

$\mathbf{A}_{p}=\left[\begin{array}{cccc}-\left(\frac{1}{\tau_{\sigma}}\right) & \left(\omega_{m}+\omega_{s l}\right) & \left(\frac{\nu_{r}}{L_{\sigma} \cdot \tau_{r}}\right) & \left(\frac{\nu_{r} \cdot \omega_{m}}{L_{\sigma}}\right) \\ \left(R_{r} \cdot \nu_{r}\right) & & \\ 0 & \left(R_{r} \cdot \nu_{r}\right) & -\left(\omega_{s l}\right) & -\left(\frac{1}{\tau_{r}}\right)\end{array}\right]$

and the variables used here are defined as follows:

$$
\begin{aligned}
& \nu_{r}=M / L_{r r} ; \quad R,=R_{s}+\nu_{r}^{2} \cdot R_{r} ; \\
& L,=L, \quad+\nu_{r} \cdot L, \quad=L_{s s}-\nu_{r} \cdot M ; \\
& \tau_{\sigma}=L_{\sigma} / R_{\sigma} ; \quad \tau_{r}=L_{r r} / R_{r}=\left(L_{\sigma r}+M\right) / R_{r}
\end{aligned}
$$

where $R$, $=$ stator resistance/phase; $R_{r}=$ rotor resistancelphase; $M=$ equivalent magnetising inductance; $L_{\sigma s}=$ stator leakage inductance; $L_{\sigma r}=$ rotor leakage inductance; $\tau_{r}=$ rotor time constant; $\omega_{s l}=$ slip speed; $\omega_{m}=$ rotor speed.

$$
\begin{aligned}
& \mathbf{B}_{p}=\left[\begin{array}{cc}
\frac{1}{L_{\boldsymbol{\sigma}}} & 0 \\
0 & \frac{1}{L_{\sigma}} \\
0 & 0 \\
0 & 0
\end{array}\right] \\
& \mathbf{C}_{p}=\left[\begin{array}{llll}
\mathbf{1} & 0 & 0 & \mathbf{0} \\
0 & 1 & 0 & 0
\end{array}\right] \\
& \mathbf{D}_{p}=\left[\begin{array}{ll}
0 & 0 \\
0 & 0
\end{array}\right]
\end{aligned}
$$

where the plant state is given by

$$
x_{p}=\left[i_{s d}, i_{s q}, \Psi_{r d}, \Psi_{r q}\right]^{T}
$$

the input to the plant is given by

$$
u_{p}=\left[V_{s d}, V_{s q}\right]^{T}
$$

(see Fig. 2) the output of the plant is given by

$$
y_{p}=\left[i_{s d}, i_{s q}\right]^{T}
$$

\subsection{Anti-alias filter dynamics and augmentation}

The plant $G_{p}(s)$, i.e. the induction motor, is a low-pass filter. As long as the sampling frequency is selected at least twice as large as the plant cutoff frequency, the effects of aliasing will be small. However, as current sensors are used to measure the stator currents, the sensors will introduce measurement noise. The high-frequency measurement noise may be aliased down to lower frequencies that are within the plant bandwidth, and thus have a detrimental effect on the system performance [6]. To avoid this, a low-pass anti-aliasing filter of the form

$$
H_{a}=\frac{a}{s+a}
$$

is inserted after the measuring devices and before the samplers. For the induction motor indicated in Appendix 7.2, the bandwidth of the system is $137 \mathrm{rad} / \mathrm{s}$. The sampling frequency is chosen as $6283.2 \mathrm{rad} / \mathrm{s}(1000 \mathrm{~Hz})$, because of limitation of the power-switching devices. 
As there are two measured outputs, i.e. $i_{s d}$ and $i_{s q}$, there will be two anti-aliasing filters of the form indicated in eqn. 5 , one for filtering $i_{s d}$ and the other for filtering $i_{s q}$. Therefore if $\mathrm{G}$, represents the anti-alias filter for both the axes, then the corresponding matrices, A, B, $\mathbf{C}$, and $\mathbf{D}$, are given by

$$
\begin{aligned}
& \mathbf{A}_{a}=\left[\begin{array}{cc}
-a & 0 \\
0 & -a
\end{array}\right] \\
& \mathbf{B}_{a}=\left[\begin{array}{ll}
a & 0 \\
0 & a
\end{array}\right] \\
& \mathbf{C}_{a}=\left[\begin{array}{ll}
1 & 0 \\
0 & 1
\end{array}\right] \\
& \mathbf{D}_{a}=\left[\begin{array}{ll}
0 & 0 \\
0 & 0
\end{array}\right]
\end{aligned}
$$

where the anti-alias filter state is given by

$$
x_{a}=\left[i_{s d a}, i_{s q a}\right]^{T}
$$

(see Fig. 2); the input to the anti-alias filter is given by

$$
u_{\alpha}=\left[\dot{i}_{s d}, \dot{i}_{s q}\right]^{T}
$$

the output of the anti-alias filter is given by

$$
y_{a}=\left[i_{s d a}, i_{s q a}\right]^{T}
$$

The plant-anti-alias filter augmented system $G_{p a}$, is described by the matrices $\mathbf{A}_{m}, \mathbf{B}_{p a}, \mathbf{C}_{p a}$ and $\mathbf{D}_{p a}$, which are given by

$$
\mathbf{A}_{p a}=\left[\begin{array}{ll}
a_{p a 11} & a_{p a 12} \\
a_{p a 21} & a_{p a 22}
\end{array}\right] \quad(6 \times 6 \text { matrix })
$$

where

$$
\begin{aligned}
& a_{p a 11}=A_{p} \\
& a_{p a 12}=0 \\
& a_{p a 21}=\left[\begin{array}{llll}
a & 0 & 0 & 0 \\
0 & a & 0 & 0
\end{array}\right] \\
& a_{p a 22}=A_{a}
\end{aligned}
$$

$$
\begin{gathered}
\mathbf{B}_{p a}=\left[\begin{array}{l}
b_{p a 11} \\
b_{p a 21}
\end{array}\right] \\
\text { where } \\
b_{p a 11}=B_{p} \\
b_{p a 21}=0
\end{gathered}
$$

and the state of $G_{p a}$ is given by

$$
x_{p a}=\left[x_{p}, x_{a}\right]^{T}
$$

the input to $G_{p a}$ is given by

the output of $G_{p a}$ is given by

$$
\begin{aligned}
& u_{p a}=u_{p} \\
& \text { given by } \\
& Y p a=Y a
\end{aligned}
$$

The augmented plant, $G_{p a}(s)$ is transformed to the discrete domain using the $\mathrm{ZOH}$ equivalent transformation to obtain $G_{p a}(z)$.

\subsection{Controller dynamics and augmentation}

Referring to Fig. 2, the unit-delay term is used to include all the computational and loop delays that may occur. This unit delay is shifted before the discrete proportional - integral control structure, to make the controller formulation easier without loss of generality.
Considering first the delay dynamics, one obtains

$$
\left[\begin{array}{c}
\varepsilon_{1 d} \\
\varepsilon_{1 q}
\end{array}\right]_{k+1}=\left[\begin{array}{l}
-i_{s d a} \\
-i_{s q a}
\end{array}\right]_{k}+\left[\begin{array}{l}
i_{s d r e f} \\
i_{s q r e f}
\end{array}\right]_{k}
$$

Considering now the proportional-integral dynamics, one obtains

$$
\left[\begin{array}{l}
\varepsilon_{2 d} \\
\varepsilon_{2 q}
\end{array}\right]_{k+1}=\left[\begin{array}{ll}
\frac{T}{2} \varepsilon_{1 d} & \varepsilon_{2 d} \\
\frac{T}{2} \varepsilon_{1 q} & \varepsilon_{2 q}
\end{array}\right]_{k}+\left[\begin{array}{c}
\frac{T}{2} \varepsilon_{1 d} \\
\frac{T}{2} \varepsilon_{1 q}
\end{array}\right]_{k+1}
$$

where $T$ is the sampling time of the analogue signals. Substituting eqn. 12 in eqn. 13, the controller dynamics $G_{c}$, is given by

$$
\begin{aligned}
{\left[\begin{array}{l}
\varepsilon_{1 d} \\
\varepsilon_{1 q} \\
\varepsilon_{2 d} \\
\varepsilon_{2 q}
\end{array}\right]_{k+1} } & {\left[\begin{array}{cccc}
0 & 0 & 0 & 0 \\
0 & 0 & 0 & 0 \\
\frac{T}{2} & 0 & 1 & 0 \\
0 & \frac{T}{2} & 0 & 1
\end{array}\right] \cdot\left[\begin{array}{c}
\varepsilon_{1 d} \\
\varepsilon_{1 q} \\
\varepsilon_{2 d} \\
\varepsilon_{2 q}
\end{array}\right]_{k} } \\
& +\left[\begin{array}{cccc}
-1 & 0 & 1 & 0 \\
0 & -1 & 0 & 1 \\
-\frac{T}{2} & 0 & \frac{T}{2} & 0 \\
0 & -\frac{T}{2} & 0 & \frac{T}{2}
\end{array}\right] \cdot\left[\begin{array}{c}
i_{s d a} \\
i_{s q a} \\
i_{s d r e f} \\
i_{s q r e f}
\end{array}\right]_{k}
\end{aligned}
$$

i.e.

where

$$
x_{c \mid k+1}=A_{c} x_{c \mid k}+B_{c} u_{c \mid k}
$$

$$
\begin{aligned}
\mathbf{A}_{c} & =\left[\begin{array}{llll}
0 & 0 & 0 & 0 \\
0 & 0 & 0 & 0 \\
\frac{T}{2} & 0 & 1 & 0 \\
0 & \frac{T}{2} & 0 & 1
\end{array}\right] \\
\mathbf{B}_{c} & =\left[\begin{array}{cccc}
-1 & 0 & 1 & 0 \\
0 & -1 & 0 & 1 \\
-\frac{T}{2} & 0 & \frac{T}{2} & 0 \\
0 & -\frac{T}{2} & 0 & \frac{T}{2}
\end{array}\right]
\end{aligned}
$$

the state of $G_{c}$ is given by

$$
x_{c}=\left[\varepsilon_{1 d}, \varepsilon_{1 q}, \varepsilon_{2 d}, \varepsilon_{2 q}\right]^{\mathrm{T}}
$$

(see Fig. 2); the input to $\mathrm{G}$, is given by

$$
u_{c}=\left[i_{s d a}, i_{s q a}, i_{s d r e f}, i_{s q r e f}\right]^{T}
$$

the output of $G$, is given by

$$
y_{c}=x_{c}
$$

Augmenting the controller $G$, to the plant-filter dynamics $G_{p a}$, the total system dynamics $\mathrm{G}$ is obtained. If the total system (plant-filter and controller) is described by the matrices $\mathbf{A}, \mathbf{B}, \mathbf{C}, \mathbf{E}$ and $\mathbf{F}$, as described in eqns. 26 and 27 , one obtains

$$
\mathbf{A}=\left[\begin{array}{ll}
a_{11} & a_{12} \\
a_{21} & a_{22}
\end{array}\right] \quad(10 \times 10 \text { matrix })
$$

where

$$
\begin{aligned}
& a_{11}=A_{p a} \\
& (6 \times 6) \\
& a_{12}=0 \\
& (6 \times 4)
\end{aligned}
$$

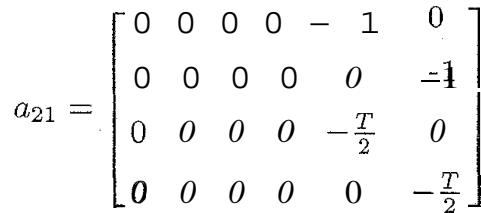

$$
\begin{aligned}
& a_{22}=A_{c}
\end{aligned}
$$




$$
\mathbf{B}=\left[\begin{array}{l}
b_{11} \\
b_{21}
\end{array}\right]
$$

where

$$
\begin{aligned}
b_{11}=B_{p a} \\
b_{21}=0 \\
\mathbf{E}=\left[\begin{array}{l}
e_{11} \\
e_{21}
\end{array}\right]
\end{aligned}
$$

where

$$
\begin{aligned}
& e_{11}=0 \\
& \text { e21 }=
\end{aligned}
$$$$
\mathbf{C}=\left[\begin{array}{llllllllll}
0 & 0 & 0 & 0 & 0 & 0 & 1 & 0 & 0 & 0 \\
0 & 0 & 0 & 0 & 0 & 0 & 0 & 1 & 0 & 0 \\
0 & 0 & 0 & 0 & 0 & 0 & 0 & 0 & 1 & 0 \\
0 & 0 & 0 & 0 & 0 & 0 & 0 & 0 & 0 & 1
\end{array}\right]
$$

$$
\mathrm{F}=\mathrm{O}
$$

The augmented total system is shown in Fig. 3, and for this system the state of $\mathrm{G}$ is given by

$$
x=\left[x_{p a}, x_{c}\right]^{T}
$$

the input to $\mathrm{G}$ is given by

$$
u=u_{p a}=u_{p}
$$

the reference input of $\boldsymbol{G}$ is given by

$$
r=\left[i_{\text {sdref }}, i_{\text {sqref }}\right]^{T}
$$

the output of $\mathrm{G}$ is given by

$$
\mathrm{Y}=y_{c}
$$

the performance output for the system is

$$
z=\left[i_{s d a}, i_{s q a}\right]^{T}=H x
$$

and the performance output matrix $\mathbf{H}$ is given by

$$
\mathbf{H}=\left[\begin{array}{llllllllll}
0 & 0 & 0 & 0 & 1 & 0 & 0 & 0 & 0 & 0 \\
0 & 0 & 0 & 0 & 0 & 1 & 0 & 0 & 0 & 0
\end{array}\right]
$$

Referring to Fig. 2, the control input to the plant-filter $u_{p a}$, is given by

$u_{p a}=\left[\begin{array}{l}V_{s d} \\ V_{s q}\end{array}\right]=-\left[\begin{array}{cccc}K_{p d} & 0 & K_{i d} & 0 \\ 0 & K_{p q} & 0 & K_{i q}\end{array}\right] \cdot\left[\begin{array}{c}\varepsilon_{1 d} \\ \varepsilon_{1 q} \\ \varepsilon_{2 d} \\ \varepsilon_{2 q}\end{array}\right]$

which is of the accepted control law $u=-K y$ where

$$
K=\left[\begin{array}{cccc}
K_{p d} & 0 & K_{i d} & 0 \\
0 & K_{p q} & 0 & K_{i q}
\end{array}\right]
$$

From eqns. $15-20$, it is evident that the induction motor is now formulated in the standard form for the LQ tracker problem, with the output feedback as indicated in eqns. 26-29. The above formulation is implemented using MATLAB.

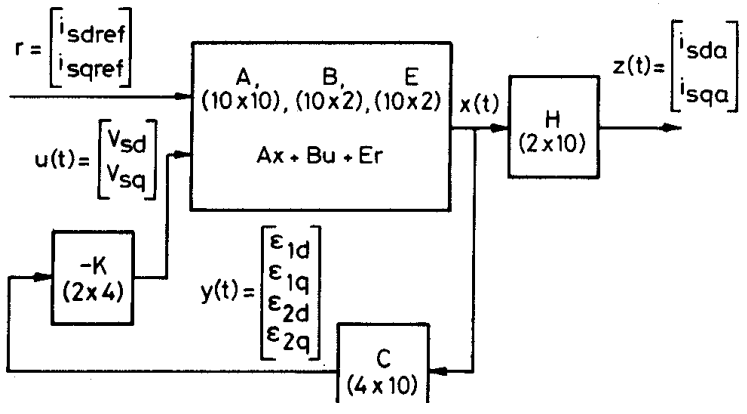

Fig.3 Augmented block diagramfor current control of induction-motordrive system with outputfeedback

3.4 Design of digital current-controller gains

The induction motor has been formulated as an LQ tracker problem with output feedback. The objective now is to determine the gain matrix $\mathbf{K}$ (with proportional and integral gains as its elements), for the above controller structure, which minimises a specified performance index. From the performance index $J$, as given in eqn. 34, one can set $V=0$ in this specific case, because the steady-state error is zero, owing to the use of the proportional - integral structure.

From eqn. 20, it is clearly seen that some of the terms in the $\mathbf{K}$ matrix are zero. Therefore in the performance index of eqn. 34, the corresponding matrix elements $k_{i j}$ can be weighted with a very large value of $g_{i j}$, so that after minimisation the resulting gain values of these $\boldsymbol{k}$, elements will be very small, and can be set to zero during implementation. This weighting of the elements of $\mathbf{K}$ has to be done for minimisation algorithms using the gradient approach [8] or the SIMPLEX approach [8]. For the SIMPLEX approach, those matrix elements which are zero can be set to zero, and the remaining matrix elements can be optimally obtained by minimisation of the performance index given by

$$
J=\frac{1}{2} \operatorname{tr}(P X)
$$

As a consequence, it would imply that with the SIMPLEX approach, one can fix any gain element of the gain matrix $\mathbf{K}$, and obtain the optimal gain values for the rest of the elements of the gain matrix $\mathbf{K}$, by minimising the performance index $J$, as given in eqn. 21 . This in fact gives lots of flexibility in arriving at the stable optimal gains of the proportional - integral control structure for the system.

The steps for obtaining the optimal gain values of the gain matrix $\mathbf{K}$, once the system is formulated as given in eqns. 15-20, is as follows:

Step I: Choice of the initial stabilising gain matrix: The optimisation process is an iterative process. Therefore, to start the optimisation process, an initial gain matrix K, which makes the closed-loop plant (Fig. 3), i.e. (A$\mathrm{BKC})$, stable, has to be used. Such a gain may or may not be easy to find. One reliable, though tedious, way to find a stabilising gain is to use discrete root locus techniques. The gain can then be optimised using the optimisation of the performance index $J$, but, using root locus techniques is rather cumbersome for systems of order greater than three. However, as the induction motor is open-loop stable (which is established from the eigenvalues of the induction-motor-system matrix), 
the compensator and the induction-motor system combination is open-loop stable. Therefore all the openloop poles lie within the unit circle. If the gains are very small, then the closed-loop poles are very close to the open-loop poles and, hence, the closed-loop system is stable. As a consequence, the proportional gains can be set to zero, and very small values of integral gains (around 0.01) can be used. This will usually provide the starting stabilising gains for the induction-motor-drive system, which can be checked by ensuring that the closed-loop eigenvalues are within the unit circle.

Step 2: Choice of state (Q) and input (R) weighting matrices: The state weighting matrix $Q$, and the input weighting matrix $\mathbf{R}$, will influence the time-domain response. Therefore they have to be suitably chosen. The state weighting matrix $\mathrm{Q}$, is equal to $H^{T} H[6]$. But to obtain good results one could use a scalar weight $q$, as follows:

$$
\mathrm{Q}=q \cdot H^{T} H
$$

If 4 is chosen as a large value, the resulting gain $K$ leads to a faster response of the system, and vice versa. Regarding the input weight matrix $R$, it is usual to choose $R=\rho_{R} I$, where $\rho_{R}$ is a scalar. If a large value of $\rho_{R}$ is used, the resulting gain $K$, leads to slower system response, and vice versa.

Step 3: Minimisation of the performance index, J: The performance index $J$, given in eqn. 21 , is minirnised under the constraint of the Lyapunov eqn. 31, using the SIMPLEX algorithm. This is done by developing an optimising routine in MATLAB, which results in optimum gain matrix $\mathbf{K}$.

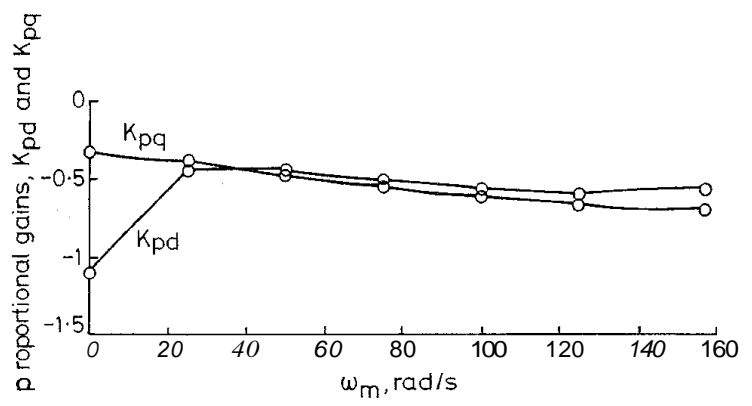

Fig.4 Optimal proportional gains versus rotor speed; $Q=0.1 H^{T} H, \boldsymbol{R}=$ diag $[0.1,2]$

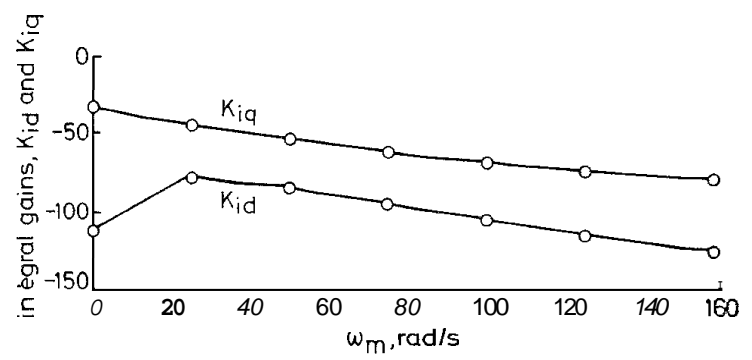

Fig.5 Optimal integral gains versus rotor speed; $Q=0.1 H^{T} H, R=$ diag $[0.1,2]$

\subsection{Simulation results}

3.5.1 Optimum gains for variable rotor speeds: For the induction motor, the plant $G_{p}$, is dependant on the rotor speed $\omega_{m}$. As a consequence, one would obtain different optimal gains at different rotor speeds. The plot of the $K_{p d}, K_{i d}, K_{p q}$ and $K_{i q}$ versus the rotor speed $\omega_{m}$ is shown in Figs. 4 and 5 , with $\mathrm{Q}=0.1 H^{T} H$ and $R=\operatorname{diag}[0.1,2]$. The gains are calculated for eight different speeds. For other speed values, the optimum values of the gains are obtained by interpolation. A lookup table can be used in the digital controller of the induction-motor-drive system, such that the optimal gain values are used at the given speed. On the other hand, one may also use a fixed-gain matrix $\mathbf{K}$, corresponding to the maximum rotor speed. This would lead to sub-optimal operation at other speed values. But, nonetheless, stability is guaranteed at all speed ranges.

3.5.2 Dynamic performance: The step responses of $i_{s d}$ and $i_{s q}$ at a speed of $\omega_{m}=157 \mathrm{rad} / \mathrm{s}$, using gains obtained with $\mathrm{Q}=0.1 H^{T} H$ and $R=\operatorname{diag}[1,20]$ are given in Figs. 6 and 7. The response $a$ is without rotor field orientation and response $b$ is with rotor field orientation. It can be clearly seen that the performance without field orientation is deteriorated.

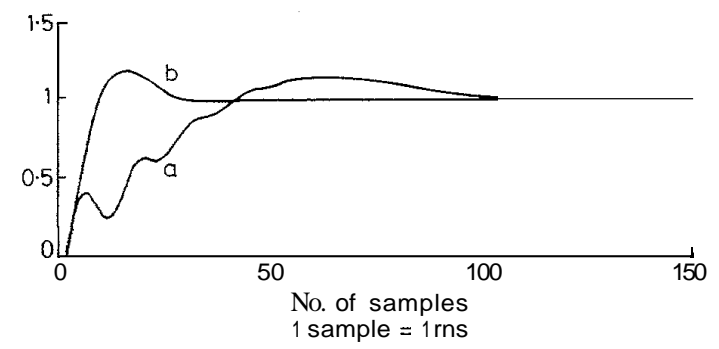

Fig. 6 Step responsefor $i_{s d}$ $b$ with rotor field orientation $\mathrm{Q}=0.1 \mathrm{H}^{\prime} \mathrm{H}, \boldsymbol{R}=\operatorname{diag}[1,20]$, Kgains $=[-0.3,-62.1088,-0.3,-48.572] ; \omega_{m}=$
$157 \mathrm{rad} / \mathrm{s}$

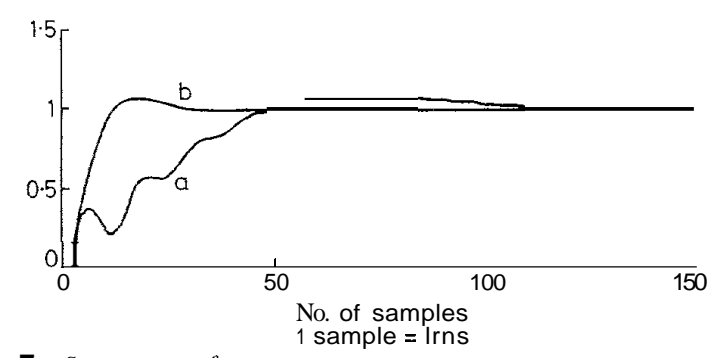

Fig.7 Step response for $i_{s q}$ $b$ with rotor field orientation

$Q=0.1 H^{\prime} H, R=\operatorname{diag}[1,20]$, Kgains $=[-0.3,-62.1088,-0.3,-48.572] ; \omega_{m}=$ $157 \mathrm{rad} / \mathrm{s}$

Under field orientation, the dynamic performance of the system can be changed by changing the $\mathbf{Q}$ and $\mathbf{R}$ matrices for finding the optimal gains. The following strategy has been used, in the context of the induction motor, for arriving at the optimal gains for different dynamic performances.

The performance index $J$, as given in eqn. 34, is very flexible, and is conducive for intuitive manipulation of the weights of the gains $g_{i j}$. The following strategy has been found useful in the context of the induction motor for arriving at the optimal gains.

- Start with the initial gain values of $K_{\text {gains }}=\left[K_{p d}, K_{i d}\right.$, $\left.K_{p q}, K_{i q}\right]=[0,-0.01,0,-0.011$

- Set $Q=H^{T} H$ and $\mathrm{R}=\operatorname{diag}(1,1)$, where the first element of the diagonal of $\mathrm{R}$ corresponds to the direct axis (d-axis) input, and the second element of the diagonal of $R$ corresponds to the quadrature axis (q-axis) input. 
- Obtain the optimal gain matrix $K$, using the SIMPLEX optimisation algorithm. This was implemented in a MATLAB environment.

- Check the step response of the closed-loop system.

- If the step response is not proper, then change the input weighting matrix $\mathbf{R}$ and the state weighting matrix $\mathbf{Q}$, and obtain $K$ until a proper response is obtained.

- If one has to slow down the system, then decrease $\mathbf{Q}$. If one has to speed up the system then increase $\mathbf{Q}$. This is shown in Figs. 8 and 9.

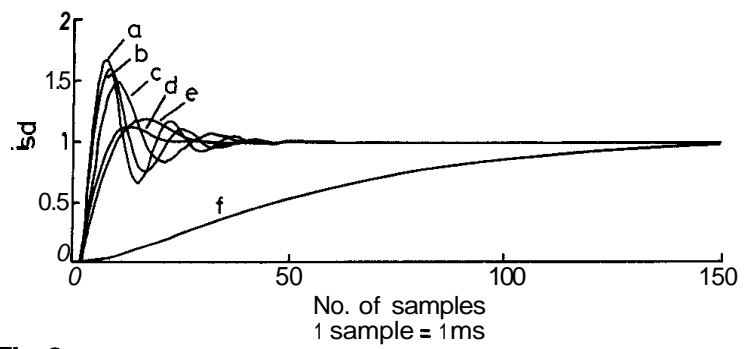

Fig.8 Step response of $i_{\text {ad }}$ under rotor field oriented control where the a, $b, c, d, e, f$ responses arefor thefollowing gains, $Q$ and $R$ $b, c, d, e, f$ responses are for thefollowing
$K$ gains $=\left[K_{p d} K_{i d} K_{p q} K_{z q}\right] ; Q ; \boldsymbol{R}=\mathrm{diag}$

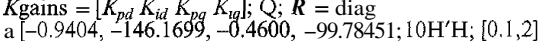

a $-0.9404,-146.1699,-0.4600,-99.78451 ; 10 \mathrm{H} H ;[0.1,2$

b $[-0.7785,-141.9508,-0.5872,-89.7422] ; \mathrm{H}^{\prime} \mathrm{H} ;[0.1,2]$

c $[-0.5457,-124.2175,-0.6775,-77.3730] ; 0.1 \mathrm{H}^{\prime} \mathrm{H} ;[0.1,2]$

$d[-0.4231,-62.1088,-0.7961,-47.9323] ; 0.1 \mathrm{H}^{\prime} \mathrm{H} ; \mathrm{l}$
$\boldsymbol{e}[-0.3,-62.1088,-0.3,-48.5720] ; 0.1 \mathrm{H}^{\prime} \mathrm{H} ;[1,20]$

$e[-0.3,-62.1088,-0.3,-48.57201 ; 0.1 \mathrm{H} H ;[1,20]$
$f[-0.0001,-5,-0.0001,-15.6961] ; \mathrm{HH} ;[1,20]$

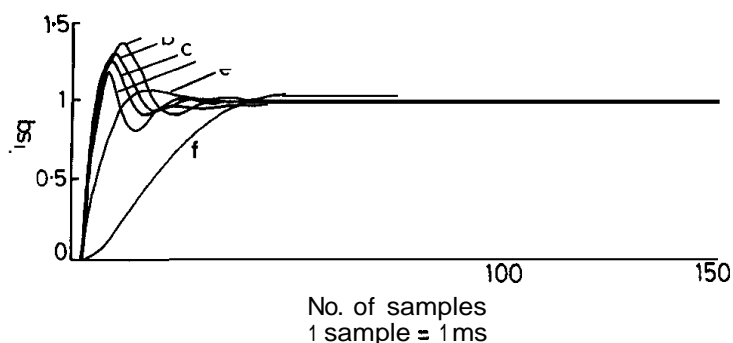

Fig.9 Step response of $i_{\text {sq }}$ under rotorfield oriented control where the a, $b, c, d, e, f$ responses are for thefollowing gains, $Q$ and $R$

$K$ gains $=\left[K_{p t} K_{i d} K_{p q} K_{i d}\right] ; \boldsymbol{Q}, \boldsymbol{R}=$ diae

a $[-0.9404,-146.1699,-0.4600,-99.7845] ; 10 \mathrm{H}^{\prime} \mathrm{H}_{;}[0.1,2]$

b $[-0.7785,-141.9508,-0.5872,-89.7422] ; \mathrm{HH} ;[0.1,2]$

c. $\left[-0.5457,-124.2175,-0.6775,-77.37301 ; 0.1 \mathrm{H}^{\prime} \mathrm{H} ;[0.1,2]\right.$

d $[-0.4231,-62.1088,-0.7961,-47.9323] ; 0.1 \mathrm{H}^{\prime} \mathrm{H} ;[1,20]$

e $[-0.3,-62.1088,-0.3,-48.5720] ; 0.1 \mathrm{H} \mathrm{H} ; 11.201$

$f[-0.0001,-5,-0.0001,-15.6961] ; \mathrm{HH} ;[1,20]$

- If one has to slow down the system controller to a large extent, then one could adopt the following method, which allows more structure to the control by way of increased intuitive interaction by the designer.

- Fix the proportional gain value, $K_{p d}$ and $K_{p q}$ to a small value. Now set the input weight matrix $\mathbf{R}$, such that $\boldsymbol{R}=\operatorname{diag}(r w d, 1)$, where $r w d$ is a very large value.

- While optimising, if gradient algorithms are used, weight the $K_{p d}$ and $K_{p q}$ gains with large weights. If the simplex algorithin is used, fix the values of $K_{p d}$ and $K_{p q}$ to low values. After optimisation, the optimal $K_{i d}$ value obtained will be a small value.

- Now set $\mathrm{R}=\operatorname{diag}(1, r w q)$, where rwq is a large weight on the q-input. Fix the $K_{i d}$ gain to the value obtained during the previous optimising run. Now the gain value, $K_{i q}$, obtained will also be low.

- $K_{i d}$ and $K_{i q}$ can be iteratively fixed and optimised to obtain any degree of slowness of speed of response.

Figs. 8 and 9 show the results of this procedure in terms of the step response of $i_{s d}$ and $i_{s q}$. It is evident that, as Q is increased, the response becomes faster. One should also note that the speed of response of $i_{s d}$ and $i_{s}$ need not be identical. This is because under rotor held-oriented control, the $i_{s d}$ component and the $i_{s q}$ components are decoupled. Further, up to base speed, the $i_{s d}$ component is kept constant, and the torque dynamics is determined only by the $i_{s q}$ component. Therefore it is sufficient that $i_{s q}$ has good dynamic performance. From Figs. 8 and 9, it is evident that response (e) is preferable from the point of view of performance criteria. In the next Section, robustness concepts are considered, along with the design methodology.

\section{Design of robust controllers for induction- motor-drive systems}

In the previous Section, it is assumed that an exact state-variable description of the plant to be controlled is available. However, in a practical situation, the actual and the model plant will never be identical. Some of the parameters, such as the rotor resistance, the stator resistance or the magnetising inductance of the actual motor, may be at variance with respect to that of the motor model, which could lead to deterioration in performance and stability. However, the design of the current controllers, considered in the context of robust controller design, accommodates for the uncertainty in the rotor resistance, stator resistance and the magnetising inductance even if parameter adaptation is not done. Further, it is often important to account for disturbances and sensor measurement noises. Disturbances may act to cause unsatisfactory performance in a system. Therefore it is important to design controllers that have stability robustness and performance robustness.

To examine the robustness properties of the system for an MIMO system, one has to use the matrix transfer relations and the singular values [6,9]. From the discussions in [6,9], it is clear that the sensor or measurement noise is generally appreciable at high frequencies. Therefore, to keep the tracking error small in the face of the measurement noise, the cosensitivity $T(j \omega)$, should be small at high frequencies above a certain frequency, say $\omega_{n}$. This may be guaranteed if

$$
\bar{\sigma}\left(G_{p a} G_{c}\right) \ll 1, \quad \text { for } \omega \geq \omega_{n}
$$

To guarantee stability robustness in the face of plantmodelling uncertainty, it can be shown [9] that the cosensitivity $T(j \omega)$ should be bounded above by the reciprocal of the multiplicative modelling discrepancy bound $m(\omega)$, which is given by

$$
\bar{\sigma}\left[G_{p a} G_{c}\left(I+G_{p a} G_{c}\right)^{-1}\right]=\bar{\sigma}(T(j \omega))<1 / m(\omega)
$$

If the worst case uncertainties in the parameters like rotor resistance, stator resistance and magnetising inductances, are known, then the upper bound $m(\omega)$ can be found as follows:

$$
\begin{aligned}
& M(j \omega)=\left(G_{p a}^{*}-G_{p a}\right) G_{p a}^{-1} \\
& m(\omega)=\bar{\sigma}(M(j \omega))
\end{aligned}
$$

where $G_{p a}^{*}$ is the actual plant and $G_{p a}$ is the model plant.

In Figs. 10-12, the robustness bound $1 / m(\omega)$, for rotor resistance, stator resistance and magnetising inductance parameter uncertainties (from $33 \%$ up to $99 \%$ ), are shown. In the case of the rotor- and statorresistance uncertainties, as the actual parameters 
increase from the nominal values (which are the cold resistance values used in the assumed model), the upper bound for $T(j \omega)$ decreases. Notice that, at higher frequencies, the system is less sensitive to uncertainty in the stator-resistance parameter than the rotor-resistance parameter. This is seen in Figs. 10 and 11, where the upper bound for stator-resistance uncertainty drops at correspondingly higher frequencies than the upper bound for the rotor-resistance uncertainty. This means that, if there is only uncertainty in the stator resistance, the permissible bandwidth of the system is higher than the case when only uncertainty in the rotor resistance exists. Although the magnetising inductance parameter uncertainty is shown up to $100 \%$ deviation from the nominal; in practice, the uncertainty in the magnetising inductance parameter will not be more than $10 \%$ to $20 \%$ [1]. One can observe from Fig. 12 that uncertainties in the magnetising inductance will also lead to lowering the permissible system bandwidth.

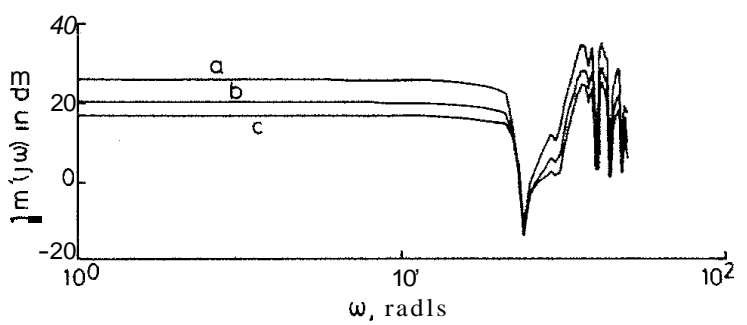

Fig.10 $1 / m(\omega)$ (robustness bounds)for rotor resistance uncertainties at $\omega_{m}=157 \mathrm{rad} / \mathrm{s}$

a $R_{r}{ }^{*}=(1.33) R_{\text {rnominal }}$

c $R_{r}^{*}=(1.99) R_{r-m o m i m a l}$

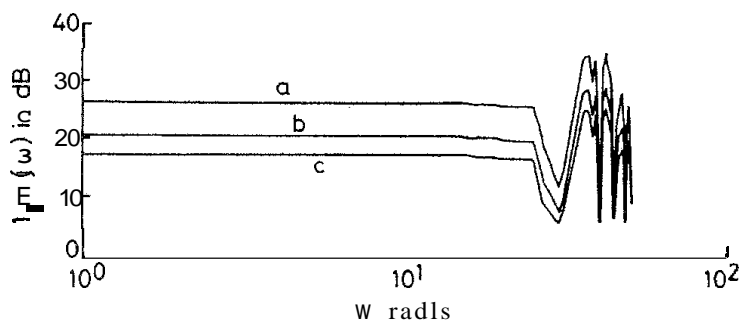

Fig.11 $1 / m(\omega)$ (robustness bounds) for stator-resistance uncertainties at $\omega_{m}=157 \mathrm{rad} / \mathrm{s}$

a $R_{s}{ }^{*}=(1.33) R_{\text {s-nominal }}$

c $R_{s}^{*}=(1.99) R_{\text {s-nomina }}$

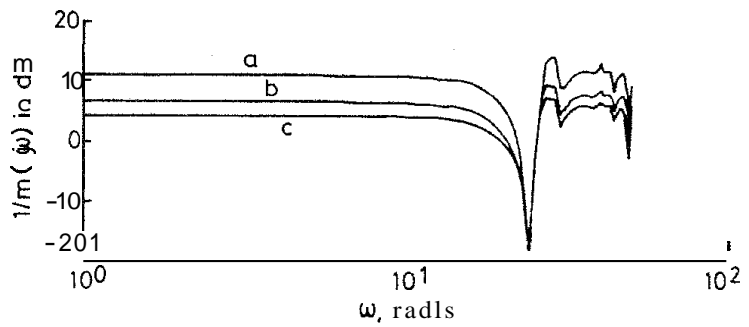

Fig.12 $I / m(\omega)$ (robustness bounds) for magnetising-inductance uncer tainties at $\omega_{m}=157 \mathrm{rad} / \mathrm{s}$

a $M^{*}=(1.33) M_{\text {nominal }}$

a $M^{*}=(1.33) M_{\text {nominal }}$
$b M^{*}=(1.66) M_{\text {nominal }}$

\subsection{Robust output-feedback controller- design algorithm}

The algorithm for the robust controller design for the induction-motor-drive system, which is formulated as an LQ tracker problem with output feedback, is as follows.
Step 1: Augment the entire drive system as an LQ tracker problem.

Step 2: Proper choices for the $\mathbf{Q}$ and the $\mathbf{R}$ matrices are made and the optimal gain matrix $\mathbf{K}$ is obtained.

Step 3: Simulate the time responses of the closed-loop system to verify that they are satisfactory. If the responses are not proper, go to step 2.

Step 4: Determine the upper bound for stability robustness. Plot the maximum singular values of $T(j \omega)$ to verify that it is within the upper bound, $1 / m(\omega)$. If it is not satisfied, return to step 2 .

\subsection{Simulation results}

The robust stability bound and the maximum singular values of $T(j \omega)$ for the response $e$ of Figs. 8 and 9 are plotted in Fig. 13. It is seen that the upper bound for guaranteed robust stability is not satisfied, as the $\bar{\sigma}(T(j \omega))$ goes beyond the upper bound at certain frequencies. Therefore, to guarantee stability robustness, one should reduce the controller bandwidth. In Fig. 14, the stability robustness bound and the maximum singular values of $T(j \omega)$ for the response $\mathbf{f}$ of Figs. 8 and 9 are plotted. It is evident that the response $f$ of Figs. 8 and 9, which is slower compared to response e, has better stability robustness. One should note that stability robustness and speed of response are always contradictory specifications. Hence, it turns out as a classic case of compromise. It is evident that, as the controller bandwidth is reduced, the system becomes more and more robust. By the same argument, it means that the field-oriented controlled induction-motor system has less stability robustness than the steady-state flux-control techniques, such as the V/f control strategies. However, field-oriented control scores over VIf control when high dynamic performance is required.
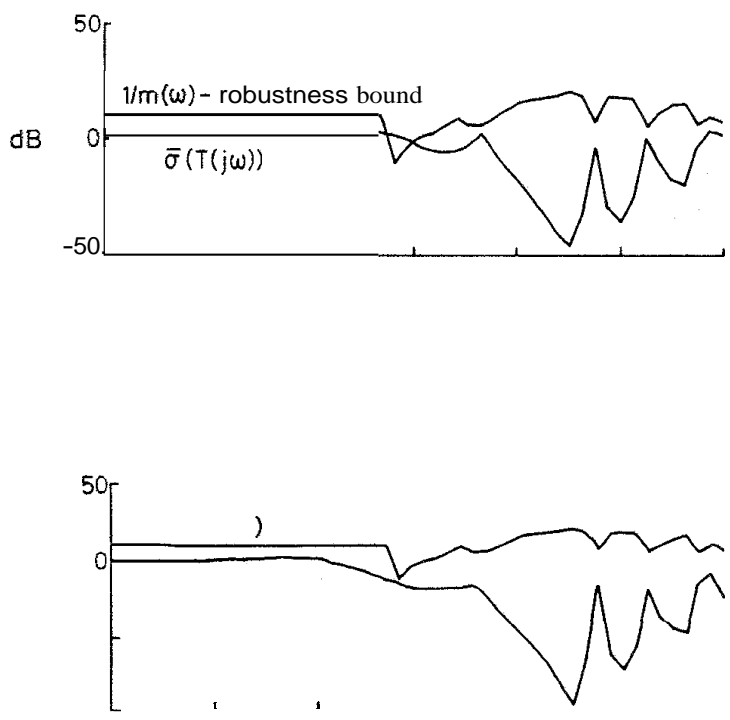

An alternative to reducing the bandwidth of the controller to improve robustness would be to adapt the parameters of the assumed model to that of the actual 


\section{Appendix}

\subsection{Summary of the discrete $L Q$ tracker with output feedback}

System Model:

$$
\begin{aligned}
& x_{k+1}=A x_{k}+B u_{k}+E r_{k} \\
& y_{k}=C x_{k}+F r_{k} \\
& z_{k}=H x_{k}
\end{aligned}
$$

where $\mathbf{x}$ is the augmented (plant + compensator) state vector, $\mathrm{u}$ is the input vector for the plant, $r$ is the reference input, $y$ is the output vector, and $z$ is the performance output which has to track the reference. The performance output need not necessarily be the same as $y$.

\section{Control:}

$$
u_{k}=-K y_{k}
$$

where $K$ is the gain matrix of the controller to be designed.

$\mathrm{dB}$

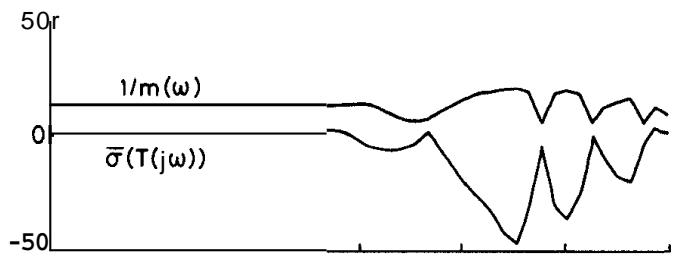

$K_{\text {gains }}$

\section{Summary}

In this paper, the induction-motor-drive system is formulated as an LQ tracker problem with output feedback. This is followed by a systematic approach to synthesis of the digital-current controllers, leading to optimal values of the gains as illustrated in the simulation results. A method of obtaining an upper bound, to guarantee the stability robustness of the system to uncertainties in the induction motor parameters, is presented. This is followed by the synthesis of robust digital-current controllers.

\section{References}

1 SEN, P.C.: 'Electric motor drives and control - past, present and future', IEEE Trans. Ind. Electron., 1990, 37, (6), pp. 562-575

2 UMANAND, L.: 'Modelling and simulation studies on parameter adaptation and digital controller synthesis for vector controlled AC drives'. Ph.D dissertation CEDT, Indian Institute of Science, India, December 1994

3 UMANAND, L., and BHAT, S.R.: 'Adaptation of the rotor time constant for the variations in the rotor resistance of an induction motor', IEEE/PESC, 1994, Vol. 1, pp. 738-743

4 UMANAND, L., and BHAT. S.R.: 'Shaft-transducerless rotor field orientation in an induction motor with stator resistance adaptation',PCIM, 1994, Nurnberg, Intelligent motion, pp. 387396

5 VAS, P.: 'Vector control of AC machines' (Clarendon Press, 1990)

6 LEWIS, F.L.: 'Applied optimal control and estimation' (Prentice Hall, 1992)

7 FRIEDLAND, B.: 'Control system design - An introduction to state space methods' (McGraw-Hill, 1987)

8 STEVENS. B.L.. VESTY. P.. HECK. B.S.. and LEWIS. F.L.: 'Loop shaping with output feedback'; Proc. American Control Conf., June 1987, pp. 146-149

9 DOYLE. J.C.. and STEIN, G.: 'Multivariable feedback design: concepts'for a classical/modern synthesis', ZEEE Trans., 1981, AC-26, (1), pp. 4-16

10 PRESS, W.H., FLANNERY, B.P., TEUKOLSKY, S.A., and VETTERLING, W.T.: 'Numerical recipes' (New York, Cambridge, 1986)
Performance index:

$$
\begin{aligned}
J= & \frac{1}{2} \sum_{k=0}^{\infty}\left(\hat{x}_{k}^{T} \cdot Q \cdot \hat{x}_{k}+\hat{u}_{k}^{T} \cdot R \cdot \hat{u}_{k}\right) \\
& +\frac{1}{2} \bar{e}^{T} \cdot V \cdot \bar{e}+\frac{1}{2} \sum_{i} \sum_{j}\left(g_{i j} \cdot k_{i j}^{2}\right)
\end{aligned}
$$

where $\hat{x}$ and $\hat{u}$ are state and input deviations, respectively, $\boldsymbol{Q}$ is the state-weighting matrix, $\boldsymbol{R}$ is the inputweighting matrix, $\bar{e}$ is the steady-state error, $V$ is the steady-state error weighting matrix and $g_{i j}$ is the weight of the gain element $k_{i j}$ of the gain matrix $K$.

Optimal output-feedback gain [I]:

$$
\begin{aligned}
& A_{c}^{T} P A_{c}-P+Q+C^{T} K^{T} R K C=0 \\
& A_{c} S A_{c}^{T}-S+X=0 \\
& R K C S C^{T}-B^{T} P A_{c} S C^{T} \\
& +B^{T}\left(A_{c}-I\right)^{-T}\left(P+H^{T} V H\right) \bar{x} \bar{y}^{T} \\
& -B^{T}\left(A_{c}-I\right)^{-T} H^{T} V r \bar{y}^{T}+g^{*} K=0
\end{aligned}
$$

where

$r_{k}$ is a step of magnitude $r$ and $g * K$ a matrix with elements $g_{i j} k_{i j}$

$\bar{x}=-\left(A_{c}-I\right)^{-1} B_{c} r$ is the state at steady state

$\bar{y}=C \bar{x}+F r$ is the output at steady state

$X=\bar{x} \cdot \bar{x}^{T}$

$\mathrm{A}_{c}=A-B K C$ is the closed loop system matrix

$\mathbf{B}_{c}=E-B K$ is the closed loop input matrix

$P$ is a positive definite constant matrix, i.e. $\boldsymbol{P}>0$

Optimal cost:

$J=\frac{1}{2} \operatorname{tr}(P X)+\frac{1}{2} \bar{e}^{T} \cdot V \cdot \bar{e}+\frac{1}{2} \sum_{i} \sum_{j}\left(g_{i j} \cdot k_{i j}^{2}\right)$

where

$$
\bar{e}=\left[I+H\left(A_{c}-I\right)^{-1} B_{c}\right] r
$$

The derivations of the eqns. 31-34 are given in [6]. 
One should note that the optimal feedback gains, $K$, are obtained by solving the set of coupled eqns. 31-33, by minimising the performance index given in eqn. 34 . This minimisation problem may be solved by using either gradient-based algorithms [10], or by non-gradient based algorithms, such as the SIMPLEX algorithm [10]. Many popular software packages, such as MATLAB, ORACLS etc., provide subroutines for the SIMPLEX minimisation algorithm. If the SIMPLEX algorithm is used, then the optimal feedback gains $K$ can be obtained by using only eqn. 31 . But, for gradient-based routines which are faster than the SIMPLEX approach, all three eqns. 31-33 are required.

\subsection{Induction-motor specifications}

3 phase, $400 \mathrm{~V} \mathrm{rms,} 50 \mathrm{~Hz}, 4$ pole machine $(p=2)$; $R_{s}=0.19 \mathrm{obms} ; R,=0.125 \mathrm{ohms} ; M=36.9 \mathrm{mH} ; L_{s s}=$ $38.51 \mathrm{mH} ; L,=37.56 \mathrm{mH}$; nominal drive torque $=$ $98 \mathrm{Nm} ; J=0.1 \mathrm{Nms}^{2} / \mathrm{rad} ; B=0.01 \mathrm{Nms} / \mathrm{rad}$. 